\title{
Semi-automatic hand annotation of egocentric recordings
}

\author{
Stijn De Beugher ${ }^{1}$, Geert Brône ${ }^{2}$, and Toon Goedemé ${ }^{1}$ \\ 1 EAVISE, ESAT - KU Leuven, Belgium \\ 2 MIDI Research Group - KU Leuven, Belgium \\ \{stijn.debeugher, Toon.Goedeme, Geert.Brone\}@kuleuven. be
}

\begin{abstract}
We present a fast and accurate algorithm for the detection of human hands in real-life 2D image sequences. We focus on a specific application of hand detection, viz. the annotation of egocentric recordings. A well known type of egocentric camera is the mobile eye-tracker, which is often used in research on human-human interaction. Nowadays, this type of data is typically annotated manually for relevant features (e.g. visual fixations of gestures), which is a time-consuming and errorprone task. We present a semi-automatic approach for the detection of human hands in images. Such an approach reduces the amount of manual analysis drastically while guaranteeing high accuracy. In our algorithm we combine several well-known detection techniques together with an advanced elimination scheme to reduce false detections. We validate our approach using a challenging dataset containing over 4300 hand instances. This validation allows us to explore the capabilities and boundaries of our approach.
\end{abstract}

Keywords: Eye-tracking, Ego-centric, Annotation, Hand detection, Humanhuman interaction, (Semi-)automatic analysis

\section{Introduction}

Our motivation for developing a highly accurate hand detector comes from the wide applicability in a variety of disciplines including computer science, linguistics, sociology and psychology. Practical applications for such a technique include human-computer and human-robot interaction, gesture detection, automatic sign language translation, active gaming, etc. Detection of human hands in real-life images is an extremely challenging task due to their varying shape, orientation and position. Recently, several highly accurate hand detection algorithms were developed for 3D images [19]. Hand detection in 2D images, however, is far from a trivial task due the lack of depth context. Several attempts were made including skin-based detections [23], model-based detections $[2,14,15]$ or pose estimation techniques [24]. Unfortunately when applied to real-life images, their performance drops significantly.

On top of the challenging task we try to tackle, we aim to develop a generic method to achieve a high detection rate. It is well known that fully automatic 
approaches typically do not guarantee high accuracy in practical cases. However many applications could benefit from such a generic approach, e.g. the removal of privacy sensitive content such as faces in mobile mapping images, generation of ground-truth data, cartography by using object detection in aerial images, etc. To overcome this we expanded our framework with an intelligent mechanism which automatically demands for manual input when the confidence of a detection is below a threshold value. Using such an approach increases the detection rate significantly at the cost of a limited amount of manual interventions. For a certain target accuracy, our system computes the minimum amount of manual interactions.

In contrast to other techniques, we focus in this work on the detection of hands in video material. Using sequences of images gives us the opportunity to use the spatio-temporal relationship between consecutive frames to increase the detection rate. We use a 3 -stage framework to generate the best possible result. First, we reduce the search space, using a human-torso detector. Second, we make a hypothesis using a sliding window approach of a hand model combined with a skin-based hand detection. Third, we use an advanced elimination approach to remove false detections in combination with a tracker resulting in reliable detections.

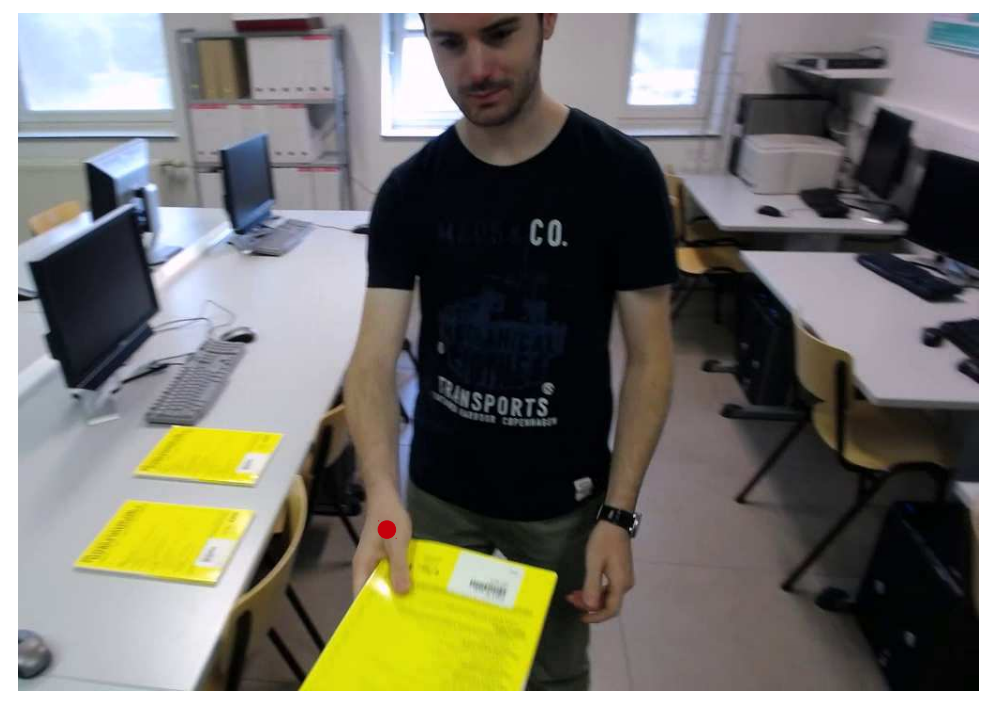

Fig. 1: Illustration of human-human interaction. Red dot is the position where the wearer of the mobile eye-tracker is looking at.

To validate our framework, we present a (semi-)automatic analysis of mobile eye-tracker data in the context of human-human interaction studies. The analysis of these data generally requires substantial manual annotation work $[11,12,1,3]$. 
The eye-tracking community would greatly benefit from the implementation of techniques that reduce the manual annotation load, like e.g. the detection of gesture strokes [11] and body language categorization [22]. The presented framework aims to contribute to these developments and proposes a technique to (semi-) automatically detect hands in video data recorded by a mobile eye-tracker. By mapping eye gaze data on interlocutors' body parts that are instrumental to face-to-face communication (like hands and faces), a first step in the analytical process is realized, as it allows for basic calculations of visual distribution. These data can then serve as the basis for further analytical work (e.g. the analysis of visual fixations on certain gesture types). An illustration of human-human interaction is given in figure 1, where an object is passed on. The red dot is the position where the giver is looking at, namely, the hand of the receiver, wearing an egocentric camera. An important research question that can be answered with this kind of experiments is if, when and how long persons look at their own hands when receiving a given object.

Next to a fast and accurate hand detection framework, an important contribution of this chapter is a generic (semi-)automatic detection approach. Furthermore, during our study, we noticed that it is hard to find fully annotated video material of human hands in real life recordings. Therefore we made our annotated dataset of eye-tracker recordings publicly available as described in [7]. The original dataset contained 1000 frames. In this paper we extended the original dataset with another challenging sequence of 1200 annotated frames. Thus the total dataset contains three sequences in which approximately 2200 frames were annotated ${ }^{3}$.

This chapter is organised as follows: In section 2, we discuss related work on hand detection. Section 3 clarifies our hand detection framework in detail. In section 4 we discuss our novel (semi-)automatic approach in which a minimal manual intervention step enhances the detection rate. Finally in section 5 we present the results on a pre-existing dataset and on our publicly available eyetracker recordings that were performed to validate the approach.

\section{Related work}

In recent years several attempts have been made to develop an accurate hand detector for 2D images, mostly by decreasing the complexity of the problem. Examples are the use of artificial markers e.g. coloured gloves [21] or using a static camera enabling the use of background segmentation [17]. In this chapter however, we focus on real-life applications where unmarked body parts need to be detected automatically, and therefore we only review the most popular methods that are applicable to natural settings.

A well known object detection technique is based on Haar-like features [20]. This technique combines a set of weak classifiers to build a final strong classifier and uses a sliding window approach to search for specific patterns in the image.

${ }^{3}$ http://www.eavise.be/insightout/Datasets/ 
In [2] this technique is used as a basis for a hand detection algorithm, in combination with a skin detector to eliminate non-hand detections. Unfortunately the performance of this technique on unconstrained images is insufficient. Newer detectors outperform greatly Haar-based techniques.

A second approach is based on the Deformable Parts Model [10], which is an extension of Histograms of Oriented Gradients (HOG) [5]. This approach allows for the definition of a model of an object which is invariant to various postures or viewing angles. In [15] this technique is used to create two models of a hand, both with and without its surrounding region, e.g. the wrist (see figure 2). In addition, they use a skin detector based on the average skin colour of the face. This skin detector is used to improve the detection rate by searching for arms in the image. Finally a super-pixel based non-maxima suppression (NMS) is used in which overlapping bounding boxes are suppressed. A drawback of this method is the high computational cost: processing a frame of $360 \times 640$ pixels takes up to 4 minutes, of which the greater part is spent on superpixel calculations. Another
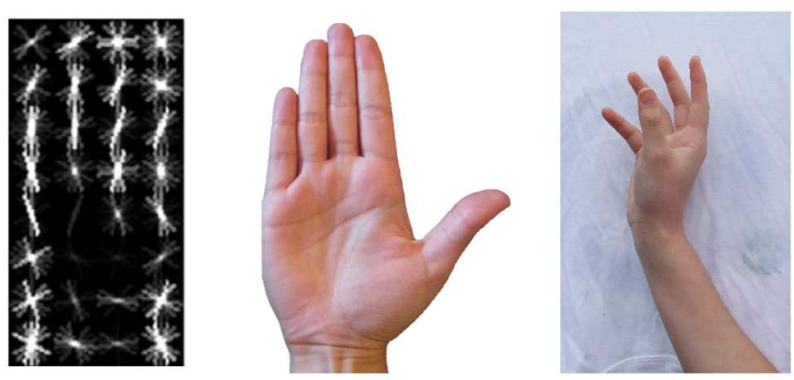

Fig. 2: Illustration of the hand models. The left image is the HOG representation of the hand model. The middle image illustrates the hand model, while the right image is an illustration of the context model (hand and its surrounding region including the background and wrist.)

hand detection approach was presented by [18]. In this chapter an invariant Hough Forest detector was used, resulting in a robust detection of the hand locations. Nevertheless, in our application the detection of the hand orientation is also of great importance on top of the location itself. Therefore we can not use such a basic approach.

In [9] the human pictorial structure is used. This approach searches for limbs in a human torso using the spatial relation between them. This method performs well on larger body parts (such as arms or heads), whereas smaller parts (e.g. a hand) are much more challenging. There are two major drawbacks of this 
method: a) the requirement that all body parts are visible in the image and b) they have a limited set of body poses that are detectable.

A pose estimation algorithm is proposed by [24]. This method is highly accurate since it has several parts for each limb and uses contextual co-occurrence relations between them. This method is designed for static images and its accuracy decreases drastically when motion blur is present, caused by moving body parts. The authors also admit their model has difficulties with some body poses (e.g. raised arms).

Based on a comparison of the previously described techniques, we opted for the work of [15] as a starting point for our algorithm. This approach achieves decent accuracy and its source code is publicly available so we can easily compare our method against it. In the next section we discuss the modifications we made in order to improve the detection results drastically, and how we extended to video.

\section{Hand Detection Framework}

An overview of our hand detection algorithm is given in figure 3 . The general idea is that we first detect a human torso in the image, giving a robust reference for the detection of smaller body parts. Next we detect the face resulting in an indication of the hand sizes. After that, we detect hands using a model introduced by [15] in combination with a skin-based detection. Then we apply an advanced elimination scheme in order to remove false detections. Finally we use a Kalman filter to track left and right hand using the spatial relationship of consecutive frames.

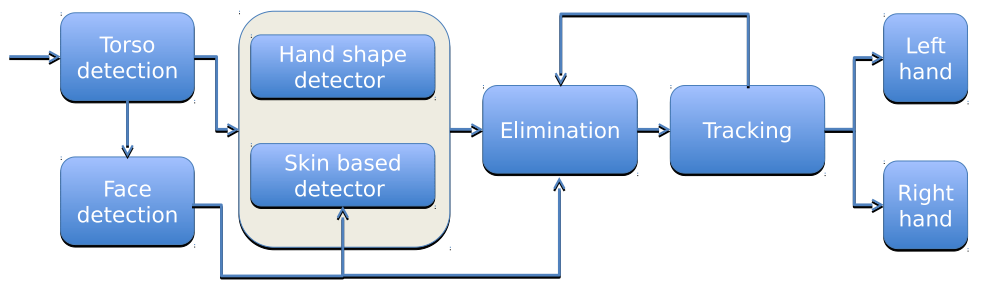

Fig. 3: Graphical representation of the proposed hand detection framework. The three stages: torso and face detection, hand detection and a combination of elimination and tracking.

\subsection{Torso Detection}

The first stage in our approach is the detection of a human torso, for which we use our own torso detector as we proposed in [6]. This torso detector is a part-based model [10], trained using only the upper $60 \%$ of the labeled bounding boxes of 
human bodies of the standard PASCAL VOC dataset ${ }^{4}$. Using this model, rather than the more widely used full person detector, has the advantage that we can cope with images in which a person is not completely visible (from head to foot) such as, for example, in most of the images captured by a mobile eye-tracker (see figure 6) in a natural setting.

\subsection{Face Detection}

The next stage is a face detection step [20], which is used as a way to further improve the accuracy of the hand detections. In the work of [15], the face detection is only used for skin segmentation. If a face is detected, they apply a skin colour based proposal method to improve their detection results. In our approach on the other hand, we also make use of the proportions of the face by rejecting hand detections which have an abnormal size compared to the size of the face. This is based on the general rule that a human face has, about the same size as an outstretched human hand.

\subsection{Hand Detection}

When the torso and face location are known, we run our actual hand detection algorithm. Instead of searching for hands in the entire image, we define a search area by expanding the torso detection bounding box in both vertical and horizontal orientation. As mentioned before, we started from the work of [15]. This means we use the same part-based deformable model of a hand, as illustrated in the left part of figure 2. In their approach, an additional context model is used. However, the experiments we ran for this study showed that the addition of this model introduces a significant amount of false detections, so that we opted not to use it.

The hand model was developed to detect upstanding hands, but in real-life recordings any hand-orientation is possible. Therefore we rotate the enlarged region around the detected torso in steps of 10 degrees per rotation, as illustrated in figure 4, yielding an accurate detection of hands in any orientation. Using a larger step size decreases the computational cost, but also affects the accuracy of the detector as shown in table 1 . This table shows the performance of the hand model on a set of 100 annotated frames of $1280 \times 720$ pixels. To further decrease the computational cost related to this type of model evaluation, we used the acceleration approach of [8].

The hand model performs well as long as a hand is clearly visible in the image. However, when a hand is not visible or strongly deformed - for example due to motion blur caused by fast movements of the arms - these models show low detection rates. To overcome this problem, we developed an additional hand detection technique as shown in figure 5. This technique segments the image in skin and no-skin based on three different colour spaces as introduced

\footnotetext{
${ }^{4}$ The PASCAL Visual Object Classes Challenge 2009 (VOC2009) Dataset http://www.pascal-network.org/challenges/VOC/voc2009/workshop/index.html
} 
Table 1: Accuracy of the hand model versus rotation angle of the images.

\begin{tabular}{c|c|c|c}
\hline Step size & Precision & Recall & Time/frame \\
\hline 10 deg. & $79,20 \%$ & $78,86 \%$ & $42 \mathrm{~s}$ \\
20 deg. & $75,78 \%$ & $75,47 \%$ & $21 \mathrm{~s}$ \\
30 deg. & $71,24 \%$ & $71,13 \%$ & $14 \mathrm{~s}$ \\
45 deg. & $62,82 \%$ & $62,55 \%$ & $9,3 \mathrm{~s}$ \\
90 deg. & $48,72 \%$ & $48,50 \%$ & $5 \mathrm{~s}$
\end{tabular}

by [16]. In this work, skin colour is defined in both Red Green Blue (RGB), Hue Saturation Value (HSV) and Luma Chroma blue Chroma red (YCbCr) colour space resulting in a robust detection mechanism for skin, even under different lighting conditions. Using this approach is an improvement compared to the work of [15], because we no longer depend on the accuracy of the face detector for skin segmentation. We apply this segmentation to the stretched torso detection as shown in figure $5(\mathrm{~b})$. Next, we skeletonize this result using a sequence of several erosion and dilation steps in order to get an accurate estimation of the skeleton, as illustrated in figure 5(c). In a following step, we apply the information obtained from the face detector. We use the correlation between the human body parts to classify the skeletonized image. If a skeletonized part has a length which is similar to the height of the face, we classify it as a hand (as illustrated by the top row in figure 5. Parts that are larger than a face are automatically treated as an arm (as illustrated by the bottom row in figure 5). For each part that is classified as an arm, we estimate a hand at both endpoints of the arm, as illustrated in figure 5(d). Estimated detections at the wrong endpoints are rejected using the elimination and tracking described in the next sections.
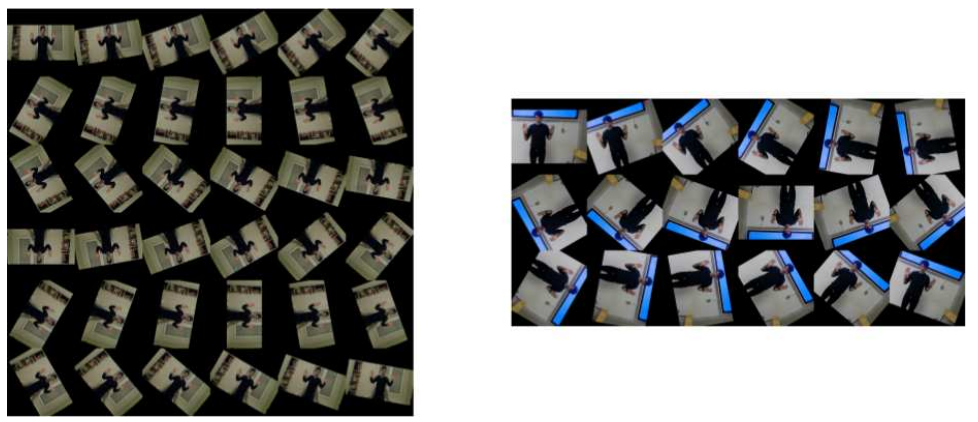

Fig. 4: Illustration of the rotation of our images in order to detect hands in any orientation. Left: step size is $10^{\circ}$ per rotation. Right: step size is $20^{\circ}$ per rotation. 


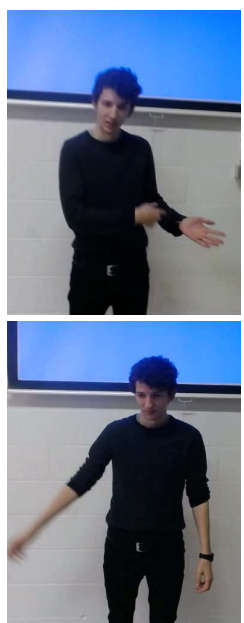

(a)
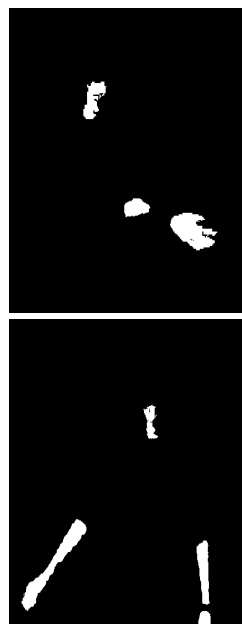

(b)

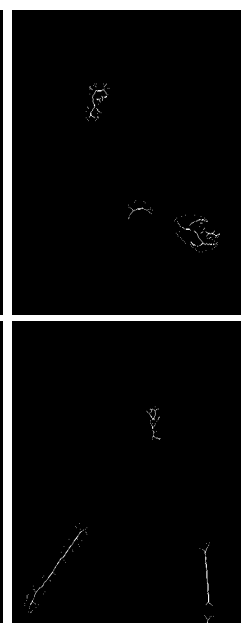

(c)

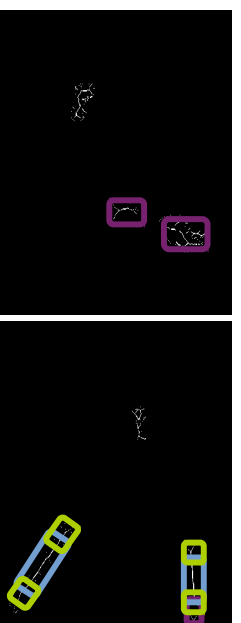

(d)

Fig. 5: From left to right: original image(a); binary image based on skin segmentation(b); skeletonization(c); arm and hand estimation(d). Purple boxes illustrate the hand classifications, blue boxes the arm detections and green boxes the estimated hands at the endpoints of the arm.

\subsection{Elimination}

After the above-mentioned steps, a large amount of hand detections is obtained, as seen in figure 6(a). The task of this elimination stage is to reject non-hand detections and to cluster overlapping detections. The output of this elimination operation is a reduced number of hand candidates as shown in figure $6(\mathrm{~b})$. In our elimination process we apply the following steps:

- Remove hand detections which have an insufficient number of skin pixels, using the same skin detection algorithm as described in the previous step.

- Remove hand detections which have a divergent size with respect to the size of the face.

- Cluster overlapping detections based on their overlap and distance between their centers.

- Reduce the contribution of clusters that coincide with the face. We noticed that a face is often detected by the hand model. Only eliminating these detections is not a viable option since persons can hold their hands in front of the face. Therefore we reduce the score of those overlapping clusters by a predefined factor to minimize the impact.

- Remove hand detections which are too far from the predicted location by the Kalman trackers.

In the elimination step, we reduced the number of hand detections. Finally we classify the remaining detections in a left and right detection using the Kalman tracker information as explained in the next section. 


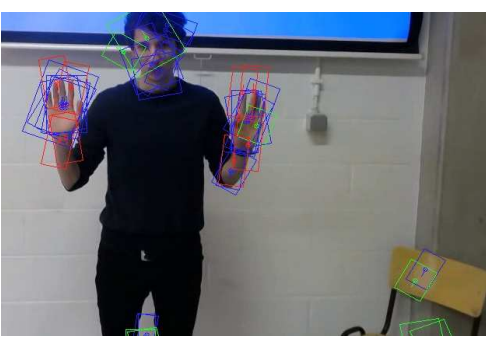

(a)

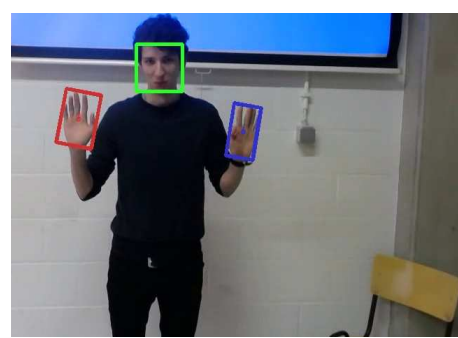

(b)

Fig. 6: Left: large amount of detections before elimination; Right: Final detections after elimination step.

\subsection{Tracking}

Our tracking stage is one of the most important contributions in order to improve the detection results. This is realized by steering the detections based on previous detections using a Kalman filter [13]. This mathematical filter is used to predict the position of the hands, which is needed when a detection is missing due to e.g. occlusions. A second advantage of using a Kalman filter is that the noise on the measured position of the detections is filtered out, resulting in more stable detections. For each torso detection we define two Kalman trackers: one for the left hand and one for the right hand in order to track each hand individually. We use a Kalman filter with the following state vector and update matrix, assuming a constant velocity motion model:

$$
\mathbf{x}=\left[\begin{array}{c}
x \\
y \\
v_{x} \\
v_{y}
\end{array}\right] \quad A=\left[\begin{array}{llll}
1 & 0 & 1 & 0 \\
0 & 1 & 0 & 1 \\
0 & 0 & 1 & 0 \\
0 & 0 & 0 & 1
\end{array}\right]
$$

where $x$ and $y$ are the position of the hand and $v_{x}$ and $v_{y}$ are the velocity of the hand. For each of the remaining clusters, as described in the previous section, we calculate the cost, based on the distance, to assign them to one of the Kalman trackers. By choosing the cluster with the lowest cost, we select the best candidate for each tracker.

To summarize this section we give an overview of our contributions as compared to the approach of [15]:

- Reduced computational footprint of our algorithm by avoiding both superpixel calculation and the validation of the context model without loss in accuracy.

- Reduced search space by using a human-torso detector and only searching for hands in a region around the torso detection. This resulted in a reduced computational time and it reduced the number of false detections.

- Skin based detection is performed even when no face is detected, resulting in more detection candidates. 
- Elimination of false-detections using the size of the face.

- Kalman tracker for both left and right hand that belongs to each torso detection.

\section{Semi-automatic analysis}

As mentioned before, we aim to develop a framework that achieves a detection rate up to $100 \%$. Obviously it is unfeasible to develop an algorithm that achieves perfect accuracy on each dataset. Therefore we expanded our hand detection framework with a generic mechanism that allows for manual intervention resulting in a much higher accuracy. The key idea is that when the confidence drops under a specific (user-defined) threshold, our algorithm requests manual input. The user then has to manually annotate the missing detection. Relying only on the detection score results in a too large amount of manual interventions. To overcome this, we also take into account the distance between a detection and the predicted position (coming from the Kalman trackers). The formula of the confidence score is shown in equation 2:

$$
M=\alpha \log \left(D_{\max }-D\right)+\beta S_{i}
$$

where:

$$
D= \begin{cases}D_{\max }-1, & \text { if } d\left(C_{i}, C_{i-1}\right) \geq D_{\max } \\ d\left(C_{i}, C_{i-1}\right), & \text { otherwise }\end{cases}
$$

$D_{\max }$ stands for the maximum allowed distance between the current detection and a detection in the previous frame, $C_{i}$ and $C_{i-1}$ define respectively the center of the current and the previous detection. $\alpha$ and $\beta$ are used to change the weight of the distance and detection score. In our experiments, we empirically determined the optimal value of those parameters: $\alpha=0.5$ and $\beta=1.0$.

The general concept of this approach is that a detection is likely to be valid if either the distance to the predicted location (based on previous detections) is low or if the detection score is high. If this value is below a user-defined threshold, manual input is requested. Thus by varying this threshold we can change the amount of manual interventions from zero (fully automatic detection) up to the number necessary to achieve full accuracy ((semi-)automatic detection). As illustrated in figure 7, the user is requested to manually annotate the missing detections when confidence score $M$ is below a certain threshold. After this manual intervention the state vector of the corresponding kalman tracker is reset, thus resulting in a stable reference point for further detections.

\section{Experimental results}

As mentioned in the introduction, we validate our hand detection framework using a data set of recordings. First we introduce our dataset, next we discuss the accuracy of our framework compared to other techniques. 


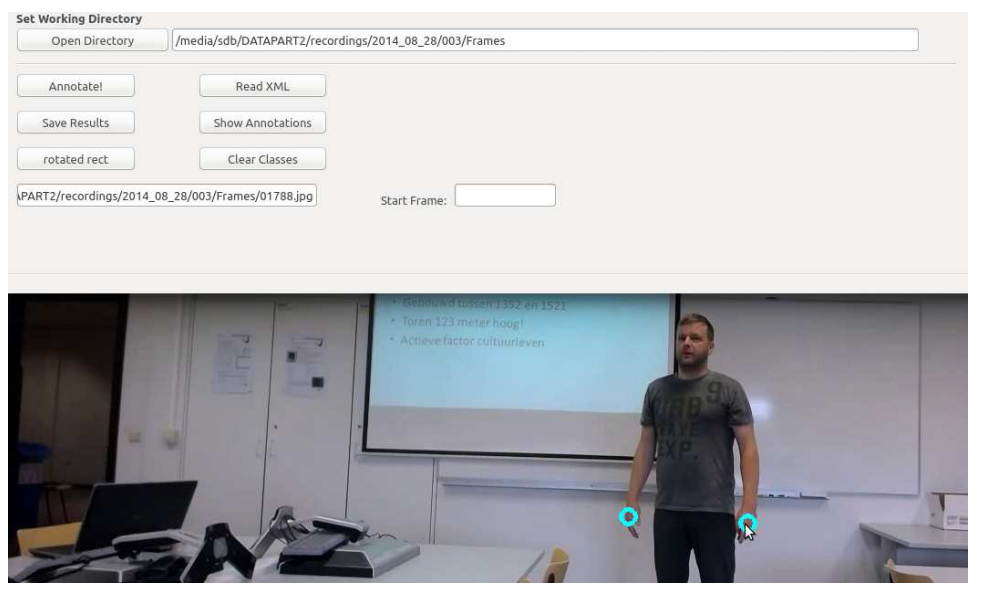

Fig. 7: Interface for manual intervention in which one can manually annotate the detection items.

\subsection{Datasets}

During our research we noticed that it is very hard to find video material containing hand annotations for each frame. In [15] a dataset of annotated movie frames is presented. Unfortunately, the available frames are not consecutive, which makes them unsuitable for our approach, designed for a sequence of frames. We also examined some video recordings from the MPI archive ${ }^{5}$, but those were annotated in terms of gestures (start and endpoint of the gesture) and contain no additional information of hand locations.

To overcome the lack of fully annotated video material, we set up a series of recordings. In each recording a mobile eye-tracker was used to capture the field of view of the test person. This eye-tracker records images at a resolution of 1280 x 720 pixels. In the first recording two persons stood face-to-face at a distance of 3 meters from each other. The person who wore the eye-tracker was told to look attentively at the interlocutor while this person made movements with his hands. The second recording was performed in a more natural setting. In this experiment, a PowerPoint presentation was given with the spectator wearing a mobile eye-tracker used as recording device. The third recording was conducted in the same setting as the second one, but another spectator was involved. This last sequence is a more challenging sequence since one of the hands are often occluded by furniture on the table. An illustration of these recordings is given in figure 8 .

For each recording we manually annotated left and right hand in a sequence of consecutive frames. The annotations of the first two sequences consist of a bounding rectangle oriented with respect to the wrist. For practical reasons, the annotations of the last sequence consist of a single point for each hand. For the

\footnotetext{
${ }^{5}$ http://corpus1.mpi.nl
} 
first sequence we chose a sequence in which a lot of arm- and hand movement is present. In total we have annotated a sequence of 403 consecutive frames. We chose a specific sequence of the second recording in which visual interaction between spectator and presenter was present, resulting in 491 consecutive frames. Finally, in the third sequence we annotated the first 1300 frames of the recording. This third sequence is an extremely difficult set for hand-tracking since the hands are often occluded by furniture. We specifically included this set since, due to its challenging nature, it fully exploits our algorithm and reveals it shortcomings.

This results in a reference dataset of 4388 annotated hand instances, which can be used as reference dataset for benchmark tests. Since it is hard to find publicly available hand-annotated video material, we made our dataset publicly available $^{6}$ for other researchers. We plan to further expand this dataset in the future.

Next to our own datasets, we also found a small publicly available dataset, viz. the '5-signers' dataset [4]. This dataset contains time-series data of the hand positions collected from 5 signers during performance of sign language. Each of the signer sequences contains 39 frames resulting in 390 annotated handinstances. An illustration of this dataset is given in the right image of figure 8 .
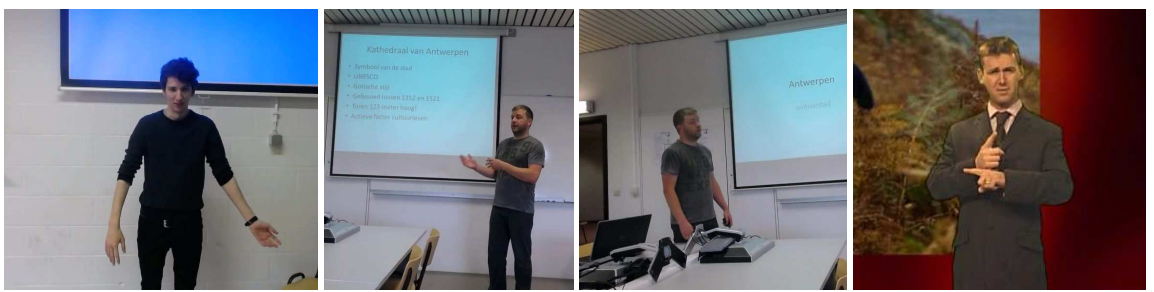

Fig. 8: Illustration of all the datasets used in this chapter. First three images are frames from our own recorded sequences, last image is a frame from the the 5-Signers dataset.

\section{$5.2 \quad$ Results}

To validate our framework, we have performed a series of experiments. First we tested our hand detection algorithm without tracking of the hands nor manual intervention. We did this experiment on the first two sequences of our own dataset and the '5-signers' dataset. Examples of the detections on those datasets are shown in figure 9 and 10 respectively. The validation is done using the Fmeasure:

$$
F=\frac{2 T P}{2 T P+F P+F N}
$$

In each frame of our sequences one person and two hands are visible. Since our framework was designed to detect two hands for each torso instance, the

\footnotetext{
${ }^{6}$ http://www.eavise.be/insightout/Datasets/
} 
number of false positives (FP) and false negatives (FN) are equal, hence the Fmeasure is reduced to the precision. A hand detection is considered valid if it is within half hand width from the ground-truth location of the hand. We compare the results to the performance of two state of the art techniques. The publicly available hand detection algorithm of [15] was used in which we use the two best detection scores as candidates for left and right hand. We also compare to the pose estimation proposal of [24] in which we classify the outermost bounding boxes of the arms as hands.

We compared our hand detection algorithm with tracking of the hands to the other techniques. Ours performs better than the other techniques in terms of accuracy. We outperfom the pose estimation technique, although a note on the bad performace of the approach of [24] should be made. The detection code we have used was developed to detect poses of persons from head to foot, whereas in the images of sequence 1 the legs of the person are not completely visible as shown in the first image of figure 8. The results of this comparison are shown in table 2. We did those experiments on our own dataset, since we need sequences of frames. It is clear that the accuracy increases significantly when the tracking is applied, as shown in the right column of table 2 .

Table 2: Accuracy of our hand detection algorithm compared to other techniques. Sequence $1 \& 2$ contains 1000 annotated hand-instances each, the '5-Signer' dataset contains 390 hand-instances.

\begin{tabular}{|c|c|c|c|c|}
\cline { 2 - 5 } \multicolumn{1}{c|}{} & Mittal $[15]$ & Yang $[24]$ & Ours & Ours incl. tracking \\
\hline Sequence 1 & $85 \%$ & $24.2 \%$ & $\mathbf{8 3 . 4 \%}$ & $\mathbf{8 8 . 2 \%}$ \\
\hline Sequence 2 & $48.9 \%$ & $46.5 \%$ & $\mathbf{5 2 . 9 \%}$ & $\mathbf{6 5 . 3 \%}$ \\
\hline 5-Signers $[4]$ & $77.6 \%$ & n.a. & $\mathbf{8 1 . 1 \%}$ & n.a. \\
\hline
\end{tabular}

We also compared the execution speed of our algorithm, as shown in table 3 . It is clear that the execution time of our algorithm is drastically lower compared to the other techniques on the same hardware (Intel Xeon E5645). Our approach is much faster compared to the work of [15] since amongst others we no longer depend on the superpixel calculation. We also outperform the computational cost of [24] by a factor of 3 .

Table 3: Execution times per frame averaged over all frames. \begin{tabular}{|l||l|}
\hline Mittal [15] Yang [24] & Ours \\
\hline
\end{tabular}

\begin{tabular}{|l|c|c|c|}
\hline Avg time/frame & $293.33 \mathrm{~s}$ & $113 \mathrm{~s}$ & $\mathbf{3 6 . 6 7} \mathbf{~ s}$ \\
\hline
\end{tabular}

Furthermore we present the extensive results of our (semi-)automatic approach on our own dataset as shown in figure 11. In this graph we plot the accuracy in function of the number of manual interventions expressed in a percent 

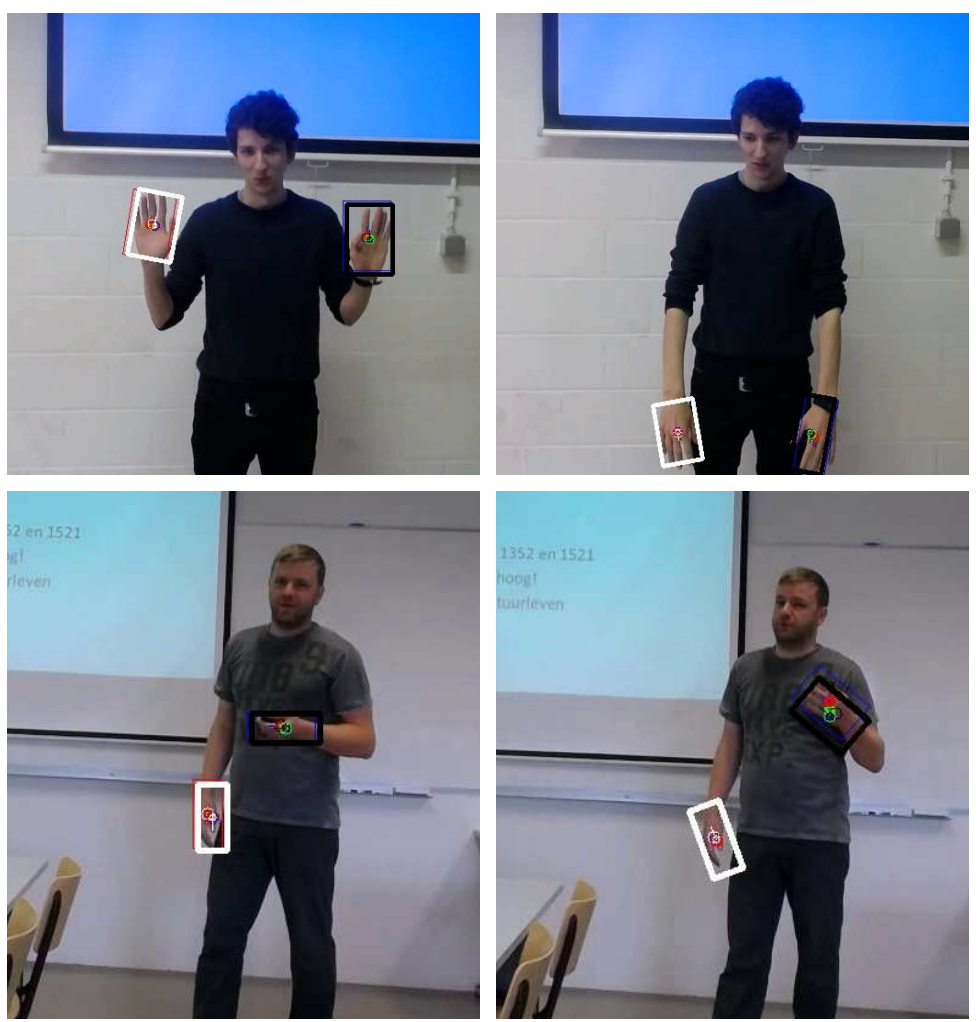

Fig. 9: Examples of hand detections on our own recorded sequences. Top row are images from our first sequence, bottom row are images from our second sequence.
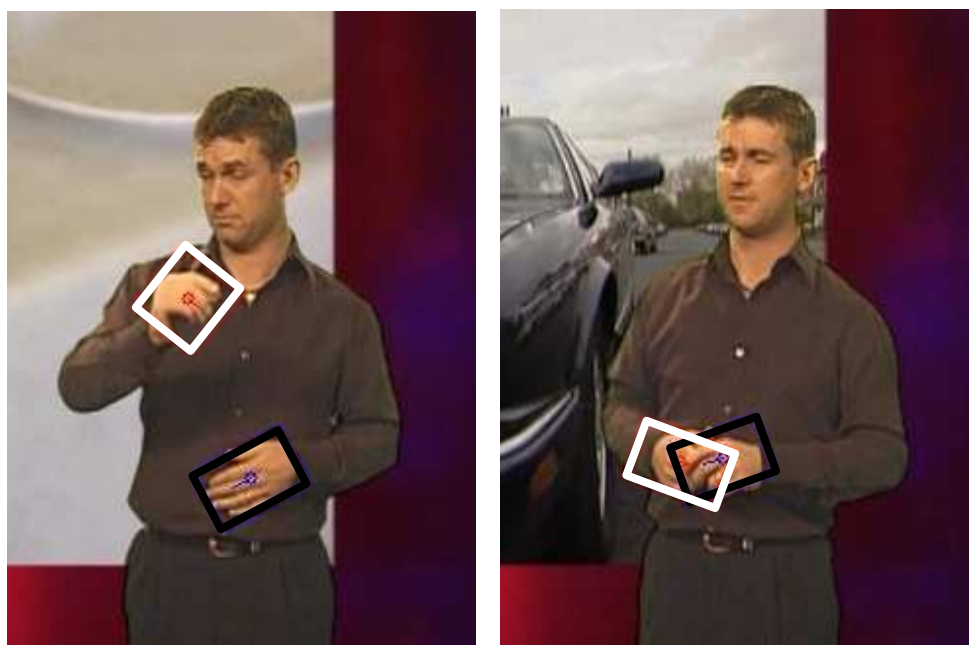

Fig. 10: Examples of hand detections on the 5-Signer dataset. 
of the numbers of frames in the sequence. As mentioned before, by thresholding the result of equation 2, we can change the amount of necessary manual interventions. It is obvious that a higher amount of manual interventions results in a higher accuracy. We should also note the improvement in accuracy between no manual intervention and the lowest amount of manual interventions. For sequence 1, the accuracy increases from $90 \%$ to $93 \%$ at the cost of only 7 manual interventions, sequence 2 on the other hand has an accuracy improvement of $12 \%$ at the cost of only 14 manual interventions. The result on sequence 3 indicates that a minimum amount of manual intervention is required in order to get a decent accuracy. This is caused by the complex setting of the recording. When we take a look at this sequence, we notice that the hands are often occluded as can be seen in figure 12. Those occlusions introduce wrong detections of the hand models. This is a perfect example in which we show the full potential of our semi-automatic approach. Without manual intervention, the accuracy on the third sequence is very bad, but when we introduce some manual intervention the accuracy increases significantly. The manual annotation of only $7.7 \%$ of the frames results in an accuracy of $94.9 \%$. We observe that each manual intervention restarts the tracker such that the hands in the following frames are again detected automatically.

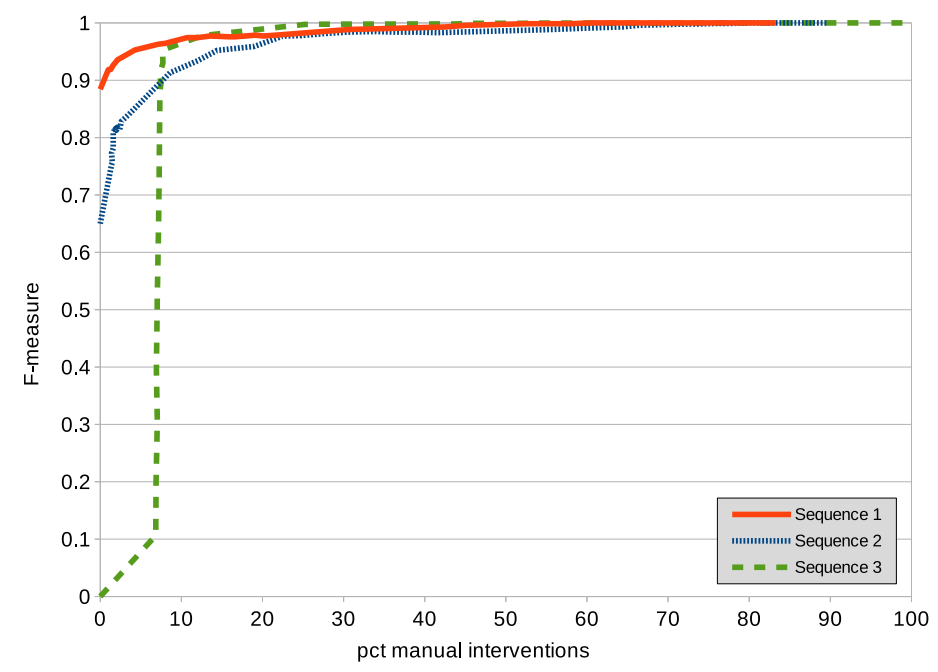

Fig. 11: Result of our (semi-)automatic approach in which accuracy is improved by manual interventions. 

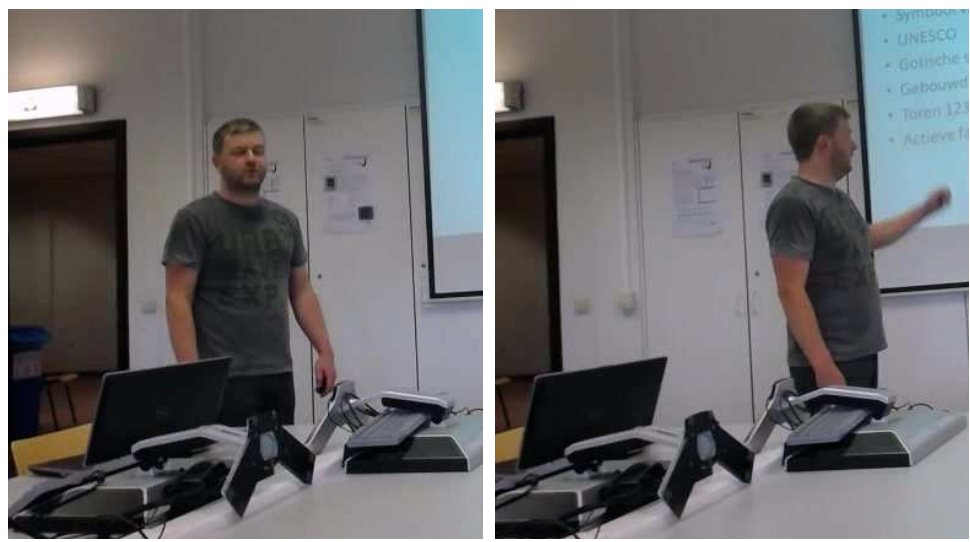

Fig. 12: Long standing occlusions in the third sequence resulting in a minimum amount of manual interventions in order to get a decent accuracy.

\section{Conclusion and Future Work}

In order to provide an alternative for the manual annotation of human hands in videos, we present a semi-automatic approach. We build on the the work of [15] and improved the accuracy and even decreased the computational cost. These improvements are realized by: a) using a torso detector to reduce the search area b) make use of a face detector whose detection is used to reject false hand detections c) integration of an accurate skin-based hand and arm detection mechanism which is especially used in images where motion blur occurs d) an advanced elimination scheme that is used to reject erroneous hand detections combined with a smart tracking mechanism for both left and right hands. We extended our approach with a generic mechanism that finds the optimal place to ask for manual intervention resulting in a much higher accuracy with minimal manual effort. This approach is based on a confidence score that is calculated using the detection score and the distance to the predicted detections. By thresholding this confidence score, we can change the amount of manual interventions. For validation we use three own recorded sequences, which we made publicly available, and one preexisting dataset. We report good accuracy and performance on several image sets as compared to state-of-the-art techniques. The third sequence is more complex than the others since hands are often occluded by furniture. When we apply our algorithm to that particular sequence, we show the full potential of our approach. The accuracy is very low without manual intervention, but when we apply a limited amount of manual interventions, the accuracy increases significantly.

Our future work concentrates on further reducing the computational cost of the hand detection algorithm. However we have realized a significant improvement in the processing time, our approach remains slow for practical use. We will investigate possibilities to reduce the computational cost of the model based detection. A first method is for example to apply a limited number of rotation 
angles around the angle of the previous detection. Next we will implement the possibility to indicate whether a hand is invisible (e.g. occluded by furniture). Such an indication could prevent that our system keeps searching for hands while no hands are visible. Furthermore we will work on the integration of the eye gaze data. Using such an approach enables the (semi-)automatic analysis of mobile eye-tracker data in terms of visual fixations on hands and reduce the manual workload related to this type of analysis.

\section{ACKNOWLEDGEMENTS}

This work is partially funded by KU Leuven via the projects Cametron and InSight Out. We also thank Raphael Den Dooven for his contributions.

\section{References}

1. Al Moubayed, S., Edlund, J., and Gustafson, J. (2013). Analysis of gaze and speech patterns in three-party quiz game interaction. In Interspeech 2013.

2. Bo, N., Dailey, M. N., and Uyyanonvara, B. (2007). Robust hand tracking in lowresolution video sequences. In Proc of the third conference on IASTED International Conference: Advances in Computer Science and Technology, pages 228-233, Anaheim, CA, USA.

3. Brône, G. and Oben, B. (2014). Insight interaction. A multimodal and multifocal dialogue corpus. In Language Resources and Evaluation.

4. Buehler, P., Everingham, M., Huttenlocher, D., and Zisserman, A. (2008). Long term arm and hand tracking for continuous sign language tv broadcasts. In Proceedings of the British Machine Vision Conference, pages 110.1-110.10. BMVA Press.

5. Dalal, N. and Triggs, B. (2005). Histograms of oriented gradients for human detection. In $C V P R$, pages 886-893.

6. De Beugher, S., Brône, G., and Goedemé, T. (2014). Automatic analysis of in-thewild mobile eye-tracking experiments using object, face and person detection. In Proc. of the 9th International Joint Conference on Computer Vision, Imaging and Computer Graphics Theory and Applications.

7. De Beugher, S., Brône, G., and Goedemé, T. (2015). Semi-automatic hand detection: a case study on real life mobile eye-tracker data. In Proc. of the 10th International Joint Conference on Computer Vision, Imaging and Computer Graphics Theory and Applications.

8. Dubout, C. and Fleuret, F. (2012). Exact acceleration of linear object detectors. In Proc. of the European Conference on Computer Vision (ECCV), pages 301-311.

9. Eichner, M., Marin-Jimenez, M., Zisserman, A., and Ferrari, V. (2012). 2D articulated human pose estimation and retrieval in (almost) unconstrained still images. International Journal of Computer Vision, 99:190-214.

10. Felzenszwalb, P. F., Girshick, R. B., McAllester, D., and Ramanan, D. (2010). Object detection with discriminatively trained part-based models. IEEE Transactions on Pattern Analysis and Machine Intelligence, 32(9):1627-1645.

11. Gebre, B. G., Wittenburg, P., and Lenkiewicz, P. (2012). Towards automatic gesture stroke detection. In the Eighth International Conference on Language Resources and Evaluation, pages 231-235. 
12. Jokinen, K. (2010). Non-verbal signals for turn-taking and feedback. In Proc. of the Seventh International Conference on Language Resources and Evaluation.

13. Kalman, R. (1960). A new approach to linear filtering and prediction problems. In Transaction of the ASME Journal of Basic Engineering, volume 82, pages 35-45.

14. Karlinsky, L., Dinerstein, M., Harari, D., and Ullman, S. (2010). The chains model for detecting parts by their context. In Computer Vision and Pattern Recognition (CVPR), 2010 IEEE Conference on, pages 25-32.

15. Mittal, A., Zisserman, A., and Torr, P. (2011). Hand detection using multiple proposals. In Proc. of the British Machine Vision Conference, pages 75.1-75.11. BMVA Press.

16. N. A. Abdul Rahim, C. W. Kit, J. S. (2006). RGB-H-CbCr skin colour model for human face detection. In MMU International Symposium on Information and Communications Technologies (M2USIC), Petaling Jaya, Malaysia.

17. Pfister, T., Charles, J., Everingham, M., and Zisserman, A. (2012). Automatic and efficient long term arm and hand tracking for continuous sign language TV broadcasts. In British Machine Vision Conference.

18. Spruyt, V., Ledda, A., and Philips, W. (2013). Real-time, long-term hand tracking with unsupervised initialization. In Proceedings of the IEEE International Conference on Image Processing, pages 3730-3734. IEEE.

19. Van den Bergh, M. and Van Gool, L. (2011). Combining rgb and tof cameras for real-time 3d hand gesture interaction. In Proceedings of the 2011 IEEE Workshop on Applications of Computer Vision (WACV), WACV'11, pages 66-72, Washington, DC, USA. IEEE Computer Society.

20. Viola, P. and Jones, M. (2001). Rapid object detection using a boosted cascade of simple features. pages 511-518. IEEE Computer Society Conference on Computer Vision and Pattern Recognition.

21. Wang, R. Y. and Popović, J. (2009). Real-time hand-tracking with a color glove. In ACM SIGGRAPH 2009 Papers, pages 63:1-63:8.

22. Williams, G., Bregler, C., Hackney, P., Rosenthal, S., Mcdowall, I., and Smolskiy, K. (2008). Body signature recognition.

23. Wu, Y., Liu, Q., and Huang, T. S. (2000). An adaptive self-organizing color segmentation algorithm with application to robust real-time human hand localization. In in Proc. of Asian Conference on Computer Vision, pages 1106-1111.

24. Yang, Y. and Ramanan, D. (2011). Articulated pose estimation with flexible mixtures-of-parts. In Computer Vision and Pattern Recognition (CVPR), 2011 IEEE Conference on, pages 1385-1392. IEEE. 